METZLER AUTORINNEN LEXIKON 


\title{
METZLER \\ AUTORINNEN \\ LEXIKON
}

\author{
Herausgegeben von \\ Ute Hechtfischer, Renate Hof, \\ Inge Stephan und Flora Veit-Wild
}

Mit 3 I9 Abbildungen

Verlag J. B. Metzler

Stuttgart - Weimar 


\title{
Inhaltsverzeichnis
}

\author{
Vorwort $\mathrm{V}$ \\ Autorinnen A-Z I-58I \\ Weiterfuhrende Bibliographie 582 \\ Verzeichnis der Mitarbeiterinnen und Mitarbeiter 596 \\ Personenregister $60 \mathrm{I}$ \\ Bildquellenverzeichnis $6 \mathrm{I} 6$
}

Die Deutsche Bibliothek - CIP-Einheitsaufnahme

\author{
Metzler-Autorinnen-Lexikon. \\ - Stuttgart ; Weimar : Metzler, I998 \\ ISBN 978-3-476-01550-1 \\ ISBN 978-3-476-03702-2 (eBook) \\ DOI $10.1007 / 978-3-476-03702-2$
}

Dieses Werk einschließlich aller seiner Teile ist urheberrechtlich geschützt.

Jede Verwertung außerhalb der engen Grenzen des Urheberrechtsgesetzes ist ohne Zustimmung des Verlages unzulässig und strafbar. Das gilt insbesondere für Vervielfältigungen, Übersetzungen, Mikroverfilmungen und die Einspeicherung und Verarbeitung in elektronischen Systemen.

(C) 1998 Springer-Verlag GmbH Deutschland

Ursprünglich erschienen bei J. B. Metzlersche Verlagsbuchhandlung und Carl Ernst Poeschel Verlag GmbH in Stuttgart 1998 


\section{Vorwort}

Von der Mystik bis zum postmodernen Experiment, von Korea bis Kanada, von Skandinavien bis Peru, von der Staatsdichterin bis zur inhaftierten Regimegegnerin - das Metzler Autorinnen Lexikon zeigt die Vielfalt weiblichen Schreibens von den Anfängen bis zur unmittelbaren Gegenwart.

Ein internationales Autorinnen-Lexikon in deutscher Sprache ist seit langem ein Desiderat. Im Metzler Autoren Lexikon, dem sich die Form unserer Texte anschließt, befindet sich unter den ca. 330 Einträgen nur eine verschwindend kleine Anzahl von Autorinnen. Das gleiche gilt für die anderen auf dem Markt befindlichen Nachschlagewerke. Während im englischsprachigen Raum schon seit Jahren mehrere Autorinnen-Lexika $\mathrm{zu}$ finden sind, existieren in Deutschland bislang nur Speziallexika. Die wenigen deutschsprachigen Nachschlagewerke, die sich ausschließlich Frauen widmen, konzentrieren sich jeweils auf einen bestimmten Zeitraum oder auf ein spezifisches Spektrum von Schriftstellerinnen.

Dagegen ist es das Anliegen dieses Lexikons, einen umfassenderen Überblick zu ermöglichen, sowohl im Hinblick auf die Anzahl der Schriftstellerinnen, als auch in bezug auf den zeitlichen Rahmen. Hierbei werden - aufgrund des internationalen Charakters dieses Nachschlagewerks - interessante Ungleichzeitigkeiten in der Entstehung und Entwicklung weiblichen Schreibens sichtbar. Für die Antike wurde die am besten überlieferte Autorin, Sappho, ausgewählt. In dieser Zeit traten auch in Asien bereits erste Schriftstellerinnen hervor. Während die Literatur von Frauen in China z. B. bis ins I. Jahrhundert. n. Chr. zurückreicht, sind in Afrika belletristische Schriftstellerinnen erst in den sechziger Jahren unseres Jahrhunderts in Erscheinung getreten. In Westeuropa dagegen gibt es eine über Jahrhunderte reichende Schreibtradition von Frauen, die von Land zu Land jedoch differierte. Es treten ähnlichen Ungleichzeitigkeiten, Verschiebungen, Überschneidungen und Beeinflussungen auf, wie sie sich auch international beobachten lassen.

Das Entstehen von schriftlichen Literaturen (im Gegensatz zur mündlichen Überlieferung) war im allgemeinen mit der Entwicklung der jeweiligen Bildungssysteme verbunden. Diese Entwicklung wiederum ist nicht unabhängig von geschlechts- und klassenspezifischen Faktoren zu betrachten, die den Zugang zu den Bildungssystemen regeln. Sie ist darüber hinaus abhängig von Ausgrenzungsmechanismen aufgrund der Zugehörigkeit zu einer bestimmten Rasse, die es zum Beispiel in den USA auch nach der Sklavenbefreiung schwarzen Frauen nahezu unmöglich machte, als Autorin anerkannt zu werden. Zudem stellt sich in den ehemaligen Kolonien überall in der Welt die Frage nach der Sprachzugehörigkeit. Vor allem die Autorinnen, die die Kolonialsprachen Englisch, Französisch, Spanisch oder Portugiesisch für ihre eigene literarische Arbeit übernommen haben, sind international bekannt geworden. Wenn wir uns in unserer Auswahl auf diese Autorinnen beschränkt haben, so sollte doch nicht übersehen werden, daß es außerdem eine Vielzahl von Schriftstellerinnen gibt, die in ihren einheimischen Sprachen schreiben; in einigen Einträgen wird auf diese Autorinnen hingewiesen. Mit anderen Worten: Die Schriftstellerinnen, die mit einem eigenen Eintrag 
in dieses Lexikon aufgenommen wurden, stehen stellvertretend für viele - für andere in einem Land, in einer Epoche, in einer literarischen Gruppierung oder in einer bestimmten Stilrichtung.

Autorinnen von belletristischen Werken bilden das Zentrum unseres Lexikons. Wir haben die Zahl auf 400 begrenzt, um ihr Leben, Werk und ihre Lebensumstände auf ein bis zwei Buchseiten möglichst anschaulich machen zu können und die Einträge nicht auf ein Gerüst von biographischen Daten reduzieren zu müssen. Aus Platzgründen konnten Autorinnen, die ausschließlich Sachbücher, Kinderbücher oder Kriminalromane geschrieben haben, nur in Ausnahmefällen aufgenommen werden. Wir haben vor allem diejenigen ausgewählt, die für die Literaturwissenschaft relevant sind, aber auch solche, auf die Leser/innen im derzeitigen Literaturangebot häufig stoßen. Das erklärt die starke Repräsentanz von Autorinnen der Gegenwart und das Übergewicht von Schriftstellerinnen aus deutschsprachigen Ländern bzw. aus Europa. Insgesamt aber enthält das Lexikon auch eine große Anzahl von Autorinnen aus afrikanischen, asiatischen und lateinamerikanischen Ländern sowie aus Neuseeland und Australien; unter ihnen viele, die in Deutschland bislang kaum bekannt geworden sind.

Die Einträge des Lexikons erheben nicht den Anspruch, bibliographisch vollständig zu sein und könnten diesen Anspruch bei über 400 aufgenommenen Autorinnen auch nicht einlösen. In die Literaturangaben unter den Beiträgen wurden nur Werkausgaben bzw. Teilsammlungen aufgenommen. Die zentralen Werke der Autorinnen sind dem Text zu entnehmen. Interessierte Leserinnen und Leser können den Literaturangaben unter den Einträgen weitere Informationen entnehmen: Dort werden - so vorhanden - auch Bibliographien genannt, vor allem aber jeweils neuere Sekundärliteratur. Um Doppelungen zu vermeiden, wurden zentrale Werke in eine allgemeine, nach Ländern bzw. Sprachräumen angeordnete Bibliographie im Anhang aufgenommen. Für den Fall, daß die Einträge keine weiterfuhhrenden Literaturangaben enthalten, sei ebenfalls auf die im Anhang genannten Standardwerke verwiesen.

Neben den namentlich verzeichneten Mitarbeiter/innen gibt es noch viele weitere, die als Spezialistinnen für die einzelnen Bereiche bei den verschiedensten Stadien der Produktion mit wertvollen Hinweisen zur Stelle waren. Unser Dank geht an Irmela Hijiya-Kirschnereit und Margarete Zimmermann, aber auch an viele andere. Besonderer Dank gebührt Carola Opitz-Wiemers, die die Auswahl und Bearbeitung der skandinavischen Autorinnen übernommen hat, Karolin Marhencke, Sabine Reihle und Jessica Hecht, die als Volontärinnen das Unternehmen im Verlag unterstützt haben und an Andrea Rupp, die die Bildbeschaffung besorgt hat.

Zum Abschluß noch einige technische Hinweise: Die Lebensdaten der vor I9I7 geborenen russischen Autorinnen werden nach dem julianischen Kalender angegeben, die entsprechenden Daten des gregorianischen Kalenders stehen in Klammern. Bei der Schreibweise von Autorinnen und Werken slawischer Sprachen wurde die wissenschaftliche Transliteration gewählt. Die Lemmata, bei denen sich unterschiedliche Schreibungen ergeben, sindy auch in transkribierter Form mit einem Verweis aufgenommen. 